\title{
Sistematização da Assistência de Enfermagem em Saúde Mental na Atenção Básica: Revisão Integrativa da Literatura
}

\section{Systematization of Nursing Assistance in Mental Health in Primary Care: Integrative Literature Review}

\author{
Lucas Marvilla Fraga de Mesquita' $\bullet$ Claudia Mara de Mello Tavares ${ }^{2}$
}

\begin{abstract}
RESUMO
O estudo objetivou descrever os conhecimentos produzidos por enfermeiros sobre o processo de enfermagem e a Sistematização da Assistência de Enfermagem - SAE relacionados à Saúde Mental no âmbito da atenção básica. A partir do uso da metodologia de Revisão Integrativa de Literatura com a questão norteadora "Qual a aplicabilidade e experiência sobre o processo de enfermagem em saúde mental na atenção básica?". Do processo de análise dos dados emergiram categorias como: "Dispositivo de cuidado no território" e "Registro de enfermagem". Evidencia-se que dispositivos extra-hospitalares de cuidado têm potência resolutiva, menos traumática e minimizadora de sofrimento estando articulado e sinérgico ao Sistema Único de Saúde (SUS). O Registro de enfermagem ainda encontra-se insuficiente ou inadequado, contudo, se expressa como dado importante e singular para construção do cuidado. Este estudo destaca como contribuições relevantes ao dar visibilidade à temática e, assim, colaborar com a compreensão de alguns aspectos relacionados à saúde da população com transtorno psíquico, auxiliando para que tanto os enfermeiros como os demais profissionais que prestam assistência a essa população possam, a partir dos achados deste estudo, refletir sobre suas práticas em saúde. Por tudo isso, as pesquisas mostraram-se conectadas aos avanços sociais e políticos, as quais asseguraram, na esfera legal, um conjunto de direitos sociais, expandindo as condições de acesso aos serviços de saúde, analisam os desafios de garantir a atenção integral à saúde e de construir estratégias efetivas de cuidado à saúde considerando necessidades, demandas e dignidade dessa população.
\end{abstract}

Palavras-chave: Processo de Enfermagem; Saúde Mental;Atenção Primária.

\begin{abstract}
The study aimed to describe the knowledge produced by nurses about the nursing process and the Systematization of Nursing Assistance - SAE related to Mental Health in the scope of basic care. From the use of the methodology of Integrative Literature Review with the guiding question "What is the applicability and experience about the process of nursing in mental health in primary care?". The process of data analysis emerged as categories such as: "Device of care in the territory" and "Registry of nursing". It is evidenced that extra-hospital care devices have a less traumatic, less traumatic and suffering-reducing power, being articulated and synergistic with the Unified Health System (SUS). The Nursing Register is still insufficient or inadequate, however, it is expressed as an important and singular data for the construction of care. This study highlights as relevant contributions in giving visibility to the thematic and, thus, collaborate with the understanding of some aspects related to the health of the population with psychic disorder, helping so that the nurses as well as the other professionals who provide assistance to this population can, the from the findings of this study, reflect on their health practices. It was concluded that the researches were connected to social and political advances, which ensured, in the legal sphere, a set of social rights, expanding the conditions of access to health services, analyze the challenges of ensuring integral health care and to construct effective strategies of health care considering the needs, demands and dignity of this population.
\end{abstract}

Keywords: Nursing Process; Mental Health; Primary Attention.

I. Enfermeiro, Professor na Universidade Iguaçu, Enfermeiro de Saúde Mental no Centro de Atenção Psicossocial III Manoel de Barros, especialista em Saúde Mental e Atenção Psicossocial, especialista em Estratégia em Saúde da Família, Mestre em Ciências do Ensino na Saúde pela Pós-graduação da Escola de Enfermagem Aurora de Afonso Costa/Universidade Federal Fluminense Niterói (RJ), Brasil. Email: lucasmfdm@gmail.com

2. Enfermeira, Pós-doutora, Professora Titular e Coordenadora Geral da Pós-graduação da Escola de Enfermagem Aurora de Afonso Costa/Universidade Federal Fluminense Niterói (RJ), Brasil. 


\section{INTRODUÇÃO}

A enfermagem baseia-se no cuidado com o ser humano e sua família, é uma área focada nas necessidades humanas e em seus níveis de assistência. A forma científica que a enfermagem utiliza em sua atuação fundamenta-se na Sistematização da Assistência de Enfermagem - SAE, na qual tem sua construção iniciada na graduação e, por fim, consolidada ao encontro da prática profissional.(I)

A atuação da enfermagem fundamenta-se na projeção e manutenção do cuidado. Desta maneira, o modo como ela se dá no cotidiano deve ser estudado e a preocupação da enfermagem em uma constante busca pela expansão e concretização do corpo de um conhecimento específico, assim como aprimorar saberes técnico-científicos, visando um cuidado respaldado nos princípios éticos, científicos e legais. ${ }^{(2)}$

A Sistematização da Assistência de Enfermagem (SAE) é uma metodologia para organizar e realizar o cuidado de enfermagem orientado por princípios científicos, a fim de evidenciar clareza, esta, conceitua-se como ferramenta organizadora do trabalho profissional quanto ao método, pessoal e instrumentos, tornando possível a operacionalização do processo de Enfermagem, que para a realidade brasileira ocorre por meio de seis fases que compreendem o histórico, o diagnóstico, o plano assistencial, o plano de cuidados ou prescrição, a evolução e o prognóstico de enfermagem. ${ }^{(3)}$

O Processo de Enfermagem é um instrumento metodológico que orienta o cuidado profissional de Enfermagem e a documentação da prática profissional, este evidencia a contribuição da Enfermagem na atenção à saúde da população, aumentando a visibilidade e o reconhecimento profissional, isto é, a essência da prática da enfermagem, o método da profissão, que auxiliam o profissional tanto na tomada de decisões quanto na prevenção e na avaliação das consequências. ${ }^{(3)}$

A Reforma Psiquiátrica Brasileira influencia(ou) na reorganização do modo de atenção da saúde mental. Nesse contexto, o cuidado de enfermagem passa a ser reorganizado e orientado para o trabalho em equipe multiprofissional. Sendo aplicado da seguinte forma: o enfermeiro deve assumir um papel de agente terapêutico, ou seja, compreender como funciona todo o processo da tecnologia de cuidado de enfermagem, e, portanto, utilizar o Processo de Enfermagem. ${ }^{(4-5)}$

Para a aplicação do Processo de Enfermagem é necessário que o enfermeiro tenha ciência: Das necessidades de saúde do sujeito, formas de abordagem e como coletar dados, organização das informações refletindo sobre o plano de cuidado, identificação e planejamento das intervenções de enfermagem, assim como uma retro avaliação da assistência prestada. ${ }^{(2)}$
Desta forma, a possibilidade do Processo de Enfermagem e o reconhecimento do enfermeiro sobre as dimensões múltiplas da concepção a respeito da saúde mental, promovem uma prática singular, humanizada e significativa, transcendendo aspectos históricos anteriores que se limitavam à oferta de alimentos, vestimenta e moradia. ${ }^{(6)}$

Destaca-se, que em saúde mental temos a necessidade de uma competência desenvolvida, a de relacionamento interpessoal, pois permite a sensibilidade do entendimento da dor do outro, compreendendo o outro a partir de seu próprio modo-referência, necessitando de investimento bilateral cliente-enfermeiro para que se obtenha sucesso no Processo de Enfermagem. ${ }^{(2-7)}$. Este estudo tem como objetivo descrever os conhecimentos produzidos por enfermeiros sobre 0 processo de enfermagem relacionado à atenção básica em saúde mental.

\section{MÉTODO}

Trata-se de uma revisão integrativa da literatura, cuja finalidade é compor critérios bem definidos sobre a coleta de dados, análise e apresentação dos resultados de pesquisa "sobre um delimitado tema ou questão, de maneira sistemática e ordenada, contribuindo para o aprofundamento do conhecimento do tema investigado". ${ }^{(8)}$

Para construção do presente estudo, foram realizadas as seguintes etapas: I) Seleção da questão norteadora; 2) Determinação dos critérios de inclusão e exclusão e busca na literatura;3) Definição das informações a serem extraídas e categorização dos estudos; 4) Avaliação dos estudos incluídos na revisão integrativa; 5) Interpretação dos resultados e; 6) A apresentação da revisão com síntese do conhecimento produzido ${ }^{(8)}$. Definiu-se como questão norteadora deste trabalho: Qual a aplicabilidade e experiência sobre o processo de enfermagem em saúde mental na atenção básica?

Os critérios adotados para inclusão dos artigos foram:Artigos relacionados ao tema Processo de Enfermagem em saúde mental na atenção básica publicados nos últimos cinco anos.

Foi realizado o levantamento dos descritores (vocabulário controlado) contidos nos Descritores em Ciência da Saúde (DeCS) e Medical Subject Heasings (MeSH) para o levantamento bibliográfico. A busca dos documentos foi realizada no mês de outubro de 2018, em três bases de dados, a saber: National Library of Medicine (PubMed); Cummulative Index to Nursing and Allied Health Literature (CINAHL) e Literatura Latino-Americana e do Caribe em Ciências da Saúde (LILACS).

Segue abaixo o demonstrativo da estratégia de busca e o volume de documentos recuperados e pré-selecionados. 
QUADRO 1 - Quantidade de artigos encontrados x estratégias de busca.

\begin{tabular}{|c|c|c|c|}
\hline \multirow{2}{*}{ 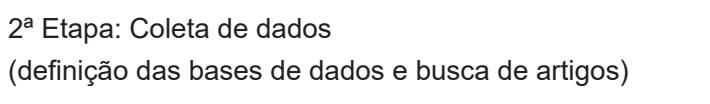 } & PubMed & CINAHL & LILACS \\
\hline & 28 & 65 & 05 \\
\hline $\begin{array}{l}3^{\text {a }} \text { Etapa: Avaliação dos dados } \\
\text { (artigos selecionados após leitura dos resumos) }\end{array}$ & 03 & 08 & 04 \\
\hline $\begin{array}{l}4^{\mathrm{a}} \text { e } 5^{\mathrm{a}} \text { Etapas: Análise, interpretação e apresentação } \\
\text { dos dados (artigos selecionados após leitura na íntegra) }\end{array}$ & 03 & 04 & 03 \\
\hline
\end{tabular}

Fonte: Mesquita, Tavares, 2018

$\mathrm{Na}$ base CINAHL foram utilizados os filtros:Texto na íntegra e recorte temporal dos últimos cinco anos. $\mathrm{Na}$ base de dados LILACS não se optou pela utilização de filtros para contemplar o máximo de artigos possíveis, visto que a utilização dos mesmos filtros trazia poucos resultados; No PubMed o filtro utilizado foi apenas o recorte temporal de cinco anos. Para recurso da busca bibliográfica usou-se o operador booleanos AND com finalidade de obter maior número de artigos possíveis que responda à questão norteadora.

\section{PERCURSSO DE SELEÇÃO DOS ESTUDOS}
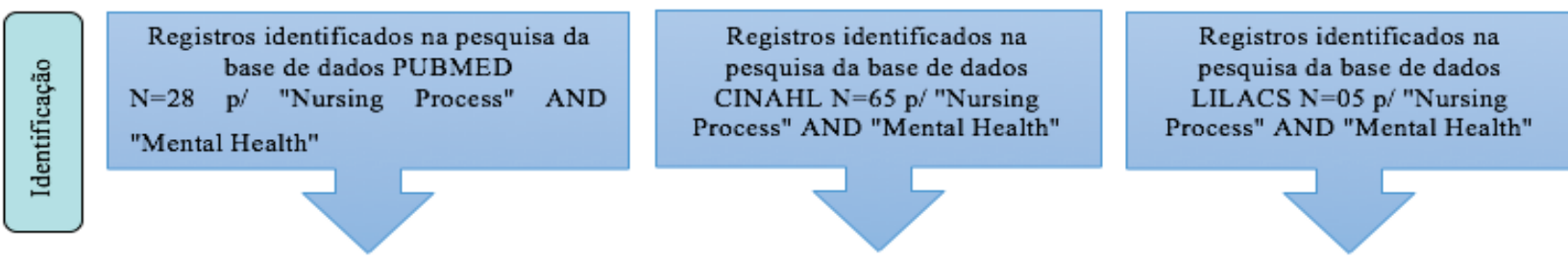

Filtro de recorte temporal 2013-2018 e artigos em português/inglês com texto completo

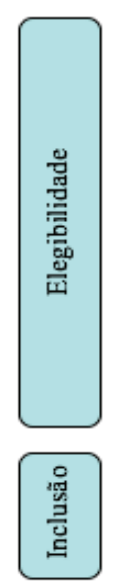

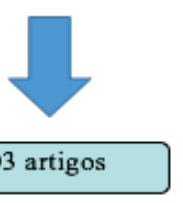

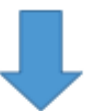

Exclusão de:

- Publicações de profissionais não enfermeiros

- Publicações sobre saúde do trabalhador

- Estudos inerentes a crianças, adolescentes e idosos, estudos que não contribuíam para a Atenção Primária

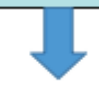

03 artigos

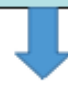

04 artigos

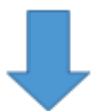

04 artigos

Leitura dos trabalhos na integra realização de fichamentos sobre o conteúdo para análise final

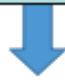

03 artigos

FIGURA 1 - Fluxograma de seleção dos estudos segundo o Preferred Reporting Items for Systematic Reviews and Meta Analyses (PRISMA)

\section{RESULTADOS}

Foram analisados 10 artigos científicos publicados em periódicos nacionais e internacionais que tinham relação com a SAE em Saúde Mental e que apresentavam dados para responder à questão norteadora do estudo. $O$ quadro apresentado a seguir mostra o material empírico utilizado para realização desta pesquisa segundo ano de publicação, título, periódico, objetivo e tipo de estudo.

\section{Análise dos dados}

Através da leitura e releitura, temas foram agregados e priorizados de acordo com categorias de maior rele- vância em relação ao objeto de estudo para serem interpretados e discutidos de acordo com o quadro teórico.

\section{Dispositivo de cuidado no território}

A SAE e o Processo de Enfermagem objetivam qualificar a assistência de enfermagem. Em ambientes com pessoas que demandam cuidados especiais torna-se uma necessidade urgente, por ser um instrumento capaz de melhorar a qualidade de vida, humanizar o cuidado e promover ações de educação em saúde..$^{(9)}$

A integração e o fornecimento de componentes eficazes de serviços de cuidado comunitário baseado em evi- 
QUADRO 1 - Distribuição dos artigos científicos segundo ano de publicação, título, periódico e objetivo, 2018

\begin{tabular}{|c|c|c|c|c|}
\hline Ano & Título & Periódico & Objetivo & Conclusão \\
\hline 2018 & $\begin{array}{l}\text { Single Therapeutic Project and Nursing Process } \\
\text { from an interdisciplinary care perspective }\end{array}$ & RGE & $\begin{array}{l}\text { Analisar o Projeto Terapêutico } \\
\text { Singular e o Processo de } \\
\text { Enfermagem quanto as suas } \\
\text { especificidades e pontos de } \\
\text { interseções, na perspectiva do } \\
\text { cuidado interdisciplinar. }\end{array}$ & $\begin{array}{l}\text { O Projeto Terapêutico Singular e o } \\
\text { Processo de Enfermagem se alinham } \\
\text { nas práticas de saúde nos serviços } \\
\text { de atenção básica e saúde mental. A } \\
\text { residência multiprofissional possibilita } \\
\text { esse alinhamento dos mesmos, e o } \\
\text { enfermeiro contribui para o } \\
\text { cuidado interdisciplinar juntamente com } \\
\text { o processo de enfermagem. }\end{array}$ \\
\hline 2017 & $\begin{array}{l}\text { Categorising Patients Mental Illnessby Medical } \\
\text { Surgical Nurses in the General Hospital Ward: A } \\
\text { Focus Group Study }\end{array}$ & ELSEVIER & $\begin{array}{l}\text { Obter informações sobre o } \\
\text { Processo de Enfermagem e } \\
\text { categorização de doenças } \\
\text { mentais em hospitais gerais. }\end{array}$ & $\begin{array}{l}\text { A linguagem utilizada pelo médico/ } \\
\text { cirurgião reflete o discurso mais amplo } \\
\text { do gerencialismo das organizações } \\
\text { de saúde. O reconhecimento dessas } \\
\text { categorias pode ser usado de forma } \\
\text { educativa, tornando um elo entre } \\
\text { serviços e decisores políticos a } \\
\text { reconsiderar a concepção de serviços } \\
\text { e oportunidades de aprendizagem para } \\
\text { enfermeiros reduzindo a estigmatização } \\
\text { de pacientes com doença mental. }\end{array}$ \\
\hline 2017 & $\begin{array}{l}\text { Nursing documentation in inpatient psychiatry: The } \\
\text { relevance of nurse-patient interactions in progress } \\
\text { notes-A focus group study with mental health staff }\end{array}$ & Wiley & $\begin{array}{l}\text { Obter informações sobre } \\
\text { a percepção da equipe de } \\
\text { saúde mental no registro das } \\
\text { evoluções em um contexto } \\
\text { da ala psiquiátrica aguda e } \\
\text { subaguda. }\end{array}$ & $\begin{array}{l}\text { Os participantes não perceberam que } \\
\text { modelo atual é baseado no processo } \\
\text { de enfermagem, apoiou-se em enfoque } \\
\text { nos recursos ou relatar interações } \\
\text { profissional-paciente. Este modelo } \\
\text { pareceu colocar os profissionais em } \\
\text { posição de especialista em relação } \\
\text { aos pacientes, tornando desafiador } \\
\text { envolver os pacientes no processo de } \\
\text { enfermagem. Aspectos essenciais de } \\
\text { cuidados de enfermagem relacionados } \\
\text { à recuperação e ao cuidado centrado na } \\
\text { pessoa não foram documentados. }\end{array}$ \\
\hline 2017 & $\begin{array}{l}\text { Saberes e fazeres no cuidado de enfermagem em } \\
\text { saúde mental }\end{array}$ & REBEn & $\begin{array}{l}\text { Compreender o cuidado } \\
\text { da enfermagem em saúde } \\
\text { mental a partir da concepção } \\
\text { de sujeito do inconsciente } \\
\text { proposta por Lacan. }\end{array}$ & $\begin{array}{l}\text { Essa perspectiva de cuidado } \\
\text { da enfermagem traz contribuições ao } \\
\text { problematizar a centralidade do sujeito e } \\
\text { da palavra nas práticas de cuidado. }\end{array}$ \\
\hline 2016 & $\begin{array}{l}\text { Processo de enfermagem na saúde mental: } \\
\text { Revisão integrativa da literatura }\end{array}$ & REBEn & $\begin{array}{l}\text { Identificar evidências da } \\
\text { literatura sobre a aplicação } \\
\text { do processo de enfermagem } \\
\text { no cuidado desenvolvido pelo } \\
\text { enfermeiro na saúde mental. }\end{array}$ & $\begin{array}{l}\text { Constatou-se movimento antagônico } \\
\text { entre cuidado pautado na relação } \\
\text { e situado na padronização de } \\
\text { diagnósticos que respondem ao } \\
\text { mal-estar físico. Verificou-se falta de } \\
\text { evidência para o uso do processo de } \\
\text { enfermagem na saúde mental e aponta- } \\
\text { se para necessidade da criação de } \\
\text { novas possibilidades de diálogo entre } \\
\text { perspectivas relacional e biológica. }\end{array}$ \\
\hline 2016 & $\begin{array}{l}\text { Integrating Evidence-BasedCommunity-Care } \\
\text { Services to Improve Schizophrenia Outcomes: A } \\
\text { Preliminary Trial }\end{array}$ & ELSEVIER & $\begin{array}{l}\text { O objetivo deste estudo } \\
\text { experimental preliminar foi } \\
\text { integrar serviços eficazes } \\
\text { de cuidados baseados em } \\
\text { evidências comunitárias que } \\
\text { são submetidas a casos } \\
\text { graves, e então examinar os } \\
\text { efeitos em indivíduos com } \\
\text { esquizofrenia. }\end{array}$ & $\begin{array}{l}\text { A satisfação dos pacientes, atitudes, } \\
\text { aceitação de medicação e níveis } \\
\text { de funcionamento geral foram } \\
\text { significativamente. Estas descobertas } \\
\text { preliminares indicam um modelo } \\
\text { potencialmente eficaz para o } \\
\text { atendimento comunitário em áreas onde } \\
\text { o gerenciamento de casos intensivo não } \\
\text { pode ser fornecido. }\end{array}$ \\
\hline 2015 & $\begin{array}{l}\text { Challenges of Documenting School children's } \\
\text { Psychosocial Health: A Qualitative Study. }\end{array}$ & SAGE & $\begin{array}{l}\text { O objetivo do estudo foi } \\
\text { explorar nas escolas de } \\
\text { enfermagem experiências } \\
\text { desafiadoras para o registro } \\
\text { da saúde psicossocial de } \\
\text { crianças escolares na Suécia. }\end{array}$ & $\begin{array}{l}\text { Enfermeiras escolares confiando } \\
\text { em sua intuição e usar um modelo } \\
\text { de documentação estruturada } \\
\text { pode aumentar as oportunidades } \\
\text { de uma documentação confiável. } \\
\text { Para desenvolver ainda mais suas } \\
\text { habilidades profissionais com } \\
\text { supervisão clínica regular podem ser } \\
\text { de grande importância. Isso, por sua } \\
\text { vez, pode aumentar as contribuições } \\
\text { para pesquisa e desenvolvimento em } \\
\text { benefício da saúde psicossocial de } \\
\text { crianças em idade escolar. }\end{array}$ \\
\hline 2014 & $\begin{array}{l}\text { Processo de Enfermagem no cotidiano do } \\
\text { enfermeiro nos Centros de Atenção Psicossocial }\end{array}$ & Revista Rene & $\begin{array}{l}\text { Compreender a realização do } \\
\text { Processo de Enfermagem no } \\
\text { cotidiano do enfermeiro que } \\
\text { atua em CAPS. }\end{array}$ & $\begin{array}{l}\text { A falta de clareza do papel do } \\
\text { enfermeiro na equipe multiprofissional } \\
\text { é um obstáculo para a elaboração } \\
\text { do projeto terapêutico individual, } \\
\text { ocasionando dificuldade na } \\
\text { incorporação do conceito de } \\
\text { integralidade e no estabelecimento } \\
\text { da relação interpessoal terapêutica, } \\
\text { para elaboração do processo de } \\
\text { Enfermagem. }\end{array}$ \\
\hline
\end{tabular}




\begin{tabular}{|l|l|l|l|l|}
\hline 2013 & $\begin{array}{l}\text { A inserção da Sistematização da Assistência } \\
\text { de Enfermagem no contexto de pessoas com } \\
\text { necessidades especiais }\end{array}$ & $\begin{array}{l}\text { Revista de } \\
\text { pesquisa } \\
\text { Cuidado é } \\
\text { Fundamental }\end{array}$ & $\begin{array}{l}\text { O objetivo foi desenvolver } \\
\text { junto aos participantes de } \\
\text { uma instituição de educação } \\
\text { especial, família e educadores } \\
\text { a Sistematização da } \\
\text { Assistência de Enfermagem } \\
\text { (SAE). }\end{array}$ & $\begin{array}{l}\text { A aplicação da SAE revelou-se como } \\
\text { um instrumento de trabalho capaz } \\
\text { de melhorar a qualidade de vida dos } \\
\text { participantes e família. }\end{array}$ \\
\hline 2013 & $\begin{array}{l}\text { Cuidado a pessoas com transtorno mental e } \\
\text { familiares: Diagnósticos e intervenções a partir da } \\
\text { consulta de enfermagem }\end{array}$ & $\begin{array}{l}\text { Descrever os diagnósticos e } \\
\text { intervenções de enfermagem } \\
\text { elaborados durante a consulta } \\
\text { de enfermagem a pessoas } \\
\text { com transtorno mental e } \\
\text { familiares em um projeto de } \\
\text { extensão universitária no ano } \\
\text { de 2010. }\end{array}$ & $\begin{array}{l}\text { Concluiu-se pela relevância da } \\
\text { aplicação de diagnósticos e } \\
\text { intervenções de enfermagem à pessoas } \\
\text { com transtornos mentais e familiares, } \\
\text { uma vez que estas intervenções } \\
\text { proporcionam ao enfermeiro um } \\
\text { cuidado direcionado, fundamentado na } \\
\text { qualidade e na excelência. }\end{array}$ \\
\hline
\end{tabular}

Fonte: Dados da pesquisa.

dências aumentam significativamente a eficácia do atendimento ao paciente com transtorno psíquico ${ }^{(10) .}$ Assim, os serviços de gerenciamento de casos (ou seja, cuidado comunitário no território) exercem influência importante sobre a medicalização e satisfação de pacientes, auxiliando os pacientes, aprimorando suas habilidades de gerenciamento de vida, identificando suas dificuldades na administração da vida diária, desenvolvendo estratégias relevantes de enfrentamento de vida (por exemplo, gerenciamento de estresse, habilidades sociais e arranjos domésticos) e adaptação à vida comunitária.

O cuidado e o processo de trabalho do enfermeiro no cenário de atenção básica foram apontados por Rocha e Lucena ${ }^{(I I)}$ como ações observacionais da saúde dos usuários, gerenciamento do trabalho e do serviço, assim como a gerência dos projetos terapêuticos e articulação dos serviços de atenção à saúde.

Ainda assim, Rocha e Lucena ${ }^{(1)}$ evidenciam o Projeto Terapêutico Singular - PTS como dispositivo eficaz para a gerência do cuidado, sobretudo, no contexto da saúde mental, descrevem que a sua implantação e os resultados são positivos nos serviços como Centros de Atenção Psicossocial (CAPS). O PTS é considerado para Rocha e Lucena ${ }^{(11)}$ uma estratégia de organização dos serviços, construído a partir da inter-relação da equipe com os outros serviços de saúde e setores da sociedade, sendo que se dá a partir das necessidades reais de cada usuário com esforço mútuo equipe-usuário-família.

Brunero, Buus e West ${ }^{(12)}$, trazem em seu artigo a experiência de internação hospitalar clínica de pacientes com transtorno psíquico, foi percebido que apesar dos diagnósticos psiquiátricos como: esquizofrenia, transtorno bipolar e depressão maior, os enfermeiros faziam separações da clientela por grandes categorias como: o gerenciado, o imprevisível, o emocional e o perigoso. As práticas descritas refletem uma abordagem de contenção moral e permanência da calmaria com foco na saúde física e uso de medicamentos para permanência e tratamento de sua doença física e sejam, portanto, "gerenciados".

Percebeu-se que para ações de cuidado em saúde mental nesta prática descrita, que só seriam evidenciadas quando algum cliente (como dito por eles) "perturbar" esse olhar evidencia, portanto, a falta de conscientização do pessoal sobre qualquer necessidade a ser explorada. ${ }^{(12)}$

Essa categorização por separação de clientela poderia ser vista por outros profissionais, como sendo pejorativo e potencialmente excludente, contudo a presença de um facilitador, um enfermeiro de saúde mental que é descrito como facilitador pode permitir a relação empática confiante para se desenvolver as ações de cuidado físico e com a saúde mental dos usuários.

\section{Registro de enfermagem}

O registro de enfermagem é uma ferramenta potencializadora do cuidado pertencente ao Processo de Enfermagem. Clausson, Berg e Janlö ${ }^{(13)}$ evidenciam que a documentação da saúde psicossocial de crianças em idade escolar no registro de saúde do aluno tem se mostrado um desafio que pode representar um risco para documentação inadequada, isto é, ocorrem transferências de informação verbal que não são registradas no prontuário dos sujeitos.

Essa informação é frequentemente relacionada à família, entendendo as razões para não documentar a saúde psicossocial de escolares, por conta do medo de interpretações erradas ou imprecisas do registro quando lidas por outras pessoas ou entidades, por se tratar de questões delicadas (como por exemplo, traços sugestivos de maus-tratos) no registro de saúde do aluno. $O$ registro possibilita $\circ$ acompanhamento progressivo do cliente visto a impossibilidade de memorização da última consulta relacionando a futuras consultas. ${ }^{(13)}$

Myklebust, Bjørkly e Råheim ${ }^{(14)}$ e Garcia et al (15) descrevem que o modelo médico domina os registros de enfermagem, compreendendo principalmente as observações dos enfermeiros sobre o comportamento dos pacientes e fornecerem apenas informações limitadas sobre as interações enfermeiro-paciente.

O foco do Processo de Enfermagem é a possibilidade de intervenções reais de melhoria na saúde dos sujeitos. A enfermagem em saúde mental se distingui por possuir como componente central, a necessidade do entendimento do processo psicoterápico a partir do estabeleci- 
mento de vínculo da relação paciente-enfermeiro. ${ }^{(7)}$

A origem dos problemas de saúde mental é multifatorial, e para dar suporte a ações terapêuticas, o enfermeiro na saúde mental contemporânea deve assumir como característica positiva do cuidado, a promoção de diversos momentos e maneiras de atuar com o sujeito no melhor sentido para sua existência, implementando o Processo de Enfermagem para tomar como forma de cuidar singular e direcionado, consolidando a atuação profissional. ${ }^{(7)}$

Sendo assim, nos pilares do cuidado, devem-se recordar os aspectos artísticos da ciência que é a enfermagem, compreendendo também um cuidado esvaziado do saber prévio, pois a assistência direta com os usuários nos convoca a valer do não saber, que não se institui de padronizações generalizantes ou generalizáveis, nos direcionando a sentir o sujeito, que demanda e demarca condições de dirigir saídas resolutivas. ${ }^{(16)}$

\section{CONCLUSÃO}

O papel do enfermeiro tem função singular no estabelecimento de vínculo para garantir um cuidado integral à saúde com os sujeitos e família, de acordo com suas reais necessidades de saúde. Nesse sentido, acredita-se que o presente estudo traz contribuições relevantes ao dar visibilidade à temática e, assim, colaborar com a compreensão de alguns aspectos relacionados à saúde da população com transtorno psíquico, auxilian- do para que tanto os enfermeiros quanto os demais profissionais que prestam assistência a essa população possam, a partir dos achados deste estudo, refletir sobre suas práticas em saúde.

Relembrando que atos sutis podem produzir efeitos de cuidado. É evidente o medo de escrever observações preconceituosas ou de interpretar mal o comportamento dos sujeitos, destaca-se, ainda, que há muitas coisas que percebemos que não podem ser provadas.

Acredita-se na relevância do presente estudo, pois possibilitou visualizar a preocupação da comunidade científica com a temática, evidenciada pelo aumento do número de trabalhos que vêm sendo desenvolvidos ao longo da última década. Destaca-se a fragilidade dos dados encontrados sobre a aplicação da SAE, os achados mostram que a literatura ainda é limitada, pois os quais não destacam as melhorias no processo de trabalho após a aplicação da SAE.

As pesquisas mostraram-se conectadas aos avanços sociais e políticos ao refletirem sobre a importância das políticas públicas específicas, as quais asseguraram, na esfera legal, um conjunto de direitos sociais, expandindo as condições de acesso aos serviços de saúde. Em contrapartida apresentam apenas modelos conceituais, experiências locais em dado contexto, ao mesmo tempo, analisam os desafios de garantir a atenção integral à saúde e de construir estratégias efetivas de cuidado à saúde considerando necessidades, demandas e dignidade dessa população. 


\section{REFERÊNCIAS}

I. Adamy K. Revista de Pesquisa: Cuidado é Fundamental Online; 3 ed. v. 5, Rio de Janeiro; 2013.

2. Brasil. Conselho Federal de Enfermagem - COFEN. Resolução $n^{\circ}$ 358/2009. [acesso em 14 de jan 2019] Disponível em: http://www.cofen.gov.br/resoluo-cofen- 3582009 4384.html.

3. Brasil. Conselho Federal de Enfermagem - COFEN. Resolução $n^{\circ}$ 159/1993 - Revogada pela Resolução Cofen $n^{\circ}$ 544/20 17. [acesso em 14 de jan 2019] Disponível em: http:// www.cofen.gov.br/resoluo-cofen-I591993_424I.html.

4. Yasui S. Rupturas e encontros: Desafios da Reforma Psiquiátrica Brasileira. Rio de Janeiro. Tese [Doutorado em Saúde Pública] - Fundação Oswaldo Cruz - FIOCRUZ; 2006.

5. Amarante P. Loucos pela Vida: a trajetória da reforma psiquiátrica no Brasil. Rio de Janeiro: SDE/ENSP; 1995.

6. Santos FOF, Montezelli JH, Peres AM. Autonomia profissional e sistematização da assistência de enfermagem: Percepção de enfermeiros. Reme - Rev. Min. Enferm. UFMG [online]. 2012 [acesso em 17 de mar 2019]; I6(2): 25 I-257. Disponível em: http://www.reme.org.br/artigo/detalhes/526.

7. Brusamarello T, Capistrano FC, Oliveira VC, Mercês NNA, Maftum MA. Cuidado a pessoas com transtorno mental e familiares: diagnósticos e intervenções a partir da consulta de enfermagem. Rev. Cogitare Enferm. UFPR [online]. 2013 [acesso em 17 mar 2019]; 18(2): 245-52. Disponível em: https://revistas.ufpr.br/cogitare/article/view/32574.

8. Mendes KDS, Silveira, RCCP; Galvão CM. Revisão Integrativa: Método de pesquisa para a incorporação de evidências na saúde e na enfermagem. Texto Contexto Enferm. [online] 2008 [acesso em 18 mar 2019]; 17(4): 758-64. Disponível em: http://www.scielo.br/pdf/tce/vI7n4//8.pdf.

9. Adamy E, Krauzer I, Hillesheim C, Silva B, Garghetti F. A inserção da sistematização da assistência de enfermagem no contexto da pessoa com necessidades especiais. Rev. Pesqui. Cuid. Fundam. [online]. 20।3; 5(3): 53-65.

10. Li JB, Liu WI, Huang MW. Integrating Evidence-Based Community-Care Services to Improve Schizophrenia Outcomes:A Preliminary Trial. Archives of Psychiatric Nursing. [online] 2016 [acesso em 19 mar 2019]; 30(I): I02-8. Dispo- nível em: https://www.ncbi.nlm.nih.gov/pubmed/268045 I0.

I I. Rocha EN, Lucena AF. Projeto Terapêutico Singular e Processo de Enfermagem em uma perspectiva de cuidado interdisciplinar. Rev. Gaúcha Enferm. [online] 2018 [acesso em 18 mar 2019]; 39: e2017-0057. Disponível em: http://www.scielo.br/pdf/rgenf/v39/I 983- I 447-rgenf-390I-e2017-0057.pdf.

12. Brunero S, Buus N, West S. Categorising Patients Mental Illness by Medical Surgical Nurses in the General Hospital Ward: A Focus Group Study. Archives of Psychiatric Nursing. [online] 2017 [acesso em 19 mar 2019]; 3I(6): 6|4-623. Disponível em: https://www.ncbi.nlm.nih.gov/pub$\mathrm{med} / 29179830$.

13. Clausson E; Berg A, Janlov AC. Challenges of Documenting Schoolchildren's Psychosocial Health: A Qualitative Study. Journal of School Nursing [online] 2014 [acesso em 18 mar 2019]; 3I (3): 205-2I I. Disponível em: https://www.researchgate.net/publication/264289508_Challenges_of_Documenting_Schoolchildren's_Psychosocial_Health_A_Qualitative_Study.

14. Myklebust K, Bjørkly S, Råheim M. Nursing documentation in inpatient psychiatry: The relevance of nurse-patient interactions in progress notes-A focus group study with mental health staff. Journal of Clinical Nursing. [online] 2018 [acesso em I 8 mar 2019]; 27(3-4): 6I I-622. Disponível em: https://www.ncbi.nlm.nih.gov/pubmed/29048775.

I5. Garcia APRF, Freitas MIP, Lamas JLT,Toledo VP. Nursing process in mental health: an integrative literature review. Rev Bras Enferm [online] 2017 [acesso em 19 mar 2019]; 70(I): 209-18. Disponível em: http://www.scielo.br/pdf/reben/ v70nI/en_0034-7I67-reben-70-0I-0220.pdf.

16. Kurimoto TCS, Penna CMM, Nitkin DIRK. Knowledge and practice in mental health nursing care. Rev Bras Enferm [online] 2017 [acesso em 19 mar 2019]; 70(5): 973-80. Disponível em: http://www.scielo.br/pdf/reben/v70n5/00347167-reben-70-05-0973.pdf.

Recebido: 2019-03-20

Aceito: 2020-02-II 